\title{
Measurement of the pseudoscalar mixing angle and $\eta^{\prime}$ gluonium content with the KLOE detector
}

The KLOE Collaboration

F. Ambrosino ${ }^{\mathrm{d}}$, A. Antonelli ${ }^{\mathrm{a}}$, M. Antonelli ${ }^{\mathrm{a}}$, C. Bacci $^{\mathrm{i}}$,

P. Beltrame ${ }^{\mathrm{b}}$, G. Bencivenni ${ }^{\mathrm{a}}$, S. Bertolucci ${ }^{\mathrm{a}}$, C. Bini ${ }^{\mathrm{g}}$, C. Bloise ${ }^{a}$, S. Bocchetta ${ }^{i}$, V. Bocci ${ }^{g}$, F. Bossi ${ }^{a}$, D. Bowring ${ }^{a, k}$,

P. Branchini ${ }^{i}$, R. Caloi ${ }^{\mathrm{g}}$, P. Campana ${ }^{\mathrm{a}}$, G. Capon ${ }^{\mathrm{a}}$,

T. Capussela ${ }^{\text {d }}$, F. Ceradini ${ }^{\text {i }}$, S. Chi ${ }^{\text {a }}$, G. Chiefari ${ }^{\mathrm{d}}$,

P. Ciambrone ${ }^{a}$, S. Conetti ${ }^{k}$, E. De Lucia ${ }^{a}$, A. De Santis ${ }^{g}$,

P. De Simone ${ }^{\mathrm{a}}$, G. De Zorzi ${ }^{\mathrm{g}}$, S. Dell'Agnello ${ }^{\mathrm{a}}$, A. Denig ${ }^{\mathrm{b}}$, A. Di Domenico ${ }^{g}$, C. Di Donato ${ }^{\mathrm{d}, 1}$, S. Di Falco ${ }^{\mathrm{e}}$, B. Di Micco ${ }^{\mathrm{i}}$, A. Doria ${ }^{\text {d }}$, M. Dreucci ${ }^{a}$, G. Felici ${ }^{a}$, A. Ferrari ${ }^{a}$, M. L. Ferrer ${ }^{\text {a }}$, G. Finocchiaro ${ }^{a}$, S. Fiore ${ }^{\mathrm{g}}$, C. Forti ${ }^{\mathrm{a}}$, P. Franzini ${ }^{\mathrm{g}}$, C. Gatti ${ }^{\mathrm{a}}$,

P. Gauzzi ${ }^{\mathrm{g}}$, S. Giovannella ${ }^{\mathrm{a}}$, E. Gorini ${ }^{\mathrm{c}}$, E. Graziani ${ }^{\mathrm{i}}$,

M. Incagli ${ }^{\text {e }}$, W. Kluge ${ }^{\mathrm{b}}$, V. Kulikov ${ }^{\mathrm{m}}$, F. Lacava $^{\mathrm{g}}$, G. Lanfranchi ${ }^{a}$, J. Lee-Franzini ${ }^{\mathrm{a}, \mathrm{j}}$, D. Leone ${ }^{\mathrm{b}}$, M. Martini ${ }^{\mathrm{a}}$, P. Massarotti ${ }^{\mathrm{d}}$, W. Mei ${ }^{\mathrm{a}}$, S. Meola ${ }^{\mathrm{d}}$, S. Miscetti ${ }^{\mathrm{a}}$, M. Moulson ${ }^{\text {a }}$, S. Müller ${ }^{\text {a }}$, F. Murtas ${ }^{\text {a }}$, M. Napolitano ${ }^{\mathrm{d}}$, F. Nguyen ${ }^{i}$, M. Palutan ${ }^{\text {a }}$, E. Pasqualucci ${ }^{\mathrm{g}}$, A. Passeri ${ }^{\mathrm{i}}$, V. Patera ${ }^{a, f}$, F. Perfetto ${ }^{d}$, L. Pontecorvo $^{g}$, M. Primavera ${ }^{c}$, P. Santangelo ${ }^{\text {a }}$, E. Santovetti ${ }^{\text {h}}$, G. Saracino ${ }^{d}$, B. Sciascia ${ }^{a}$, A. Sciubba ${ }^{\text {a,f }}$, F. Scuri ${ }^{\text {e }}$, I. Sfiligoi ${ }^{\text {a }}$, T. Spadaro ${ }^{a}$, M. Testa $^{\mathrm{g}}$, L. Tortora ${ }^{\mathrm{i}}$, P. Valente ${ }^{\mathrm{g}}$, B. Valeriani ${ }^{\mathrm{b}}$, G. Venanzoni ${ }^{\mathrm{a}}$, S. Veneziano ${ }^{\mathrm{g}}$, A. Ventura $^{\mathrm{c}}$, R.Versaci ${ }^{\mathrm{a}}, \mathrm{G} . \mathrm{Xu}^{\mathrm{a}, \ell}$

${ }^{a}$ Laboratori Nazionali di Frascati dell'INFN, Frascati, Italy.

${ }^{\mathrm{b}}$ Institut für Experimentelle Kernphysik, Universität Karlsruhe, Germany.

${ }^{\mathrm{c}}$ Dipartimento di Fisica dell'Università e Sezione INFN, Lecce, Italy.

${ }^{\mathrm{d}}$ Dipartimento di Scienze Fisiche dell'Università "Federico II" e Sezione INFN, Napoli, Italy

e Dipartimento di Fisica dell'Università e Sezione INFN, Pisa, Italy.

${ }^{\mathrm{f}}$ Dipartimento di Energetica dell'Università "La Sapienza", Roma, Italy. 


\title{
"Dipartimento di Fisica dell'Università "La Sapienza" e Sezione INFN, Roma, Italy. \\ h Dipartimento di Fisica dell'Università “Tor Vergata" e Sezione INFN, Roma, Italy. \\ i Dipartimento di Fisica dell'Università "Roma Tre" e Sezione INFN, Roma, Italy. \\ ${ }^{j}$ Physics Department, State University of New York at Stony Brook, USA. \\ ${ }^{\mathrm{k}}$ Physics Department, University of Virginia, USA. \\ ${ }^{\ell}$ Permanent address: Institute of High Energy Physics of Academica Sinica, Beijing, China. \\ ${ }^{\mathrm{m}}$ Permanent address: Institute for Theoretical and Experimental Physics, Moscow, Russia.
}

\begin{abstract}
We have measured the ratio $R_{\phi}=\mathrm{BR}\left(\phi \rightarrow \eta^{\prime} \gamma\right) / \mathrm{BR}(\phi \rightarrow \eta \gamma)$ by looking for the radiative decays $\phi \rightarrow \eta^{\prime} \gamma$ and $\phi \rightarrow \eta \gamma$ into the final states $\pi^{+} \pi^{-} 7 \gamma$ and $7 \gamma$, respectively, in a sample of $\sim 1.4 \times 10^{9} \phi$ mesons produced at the Frascati $\phi$ factory. We obtain $R_{\phi}=\left(4.77 \pm 0.09_{\text {stat }} \pm 0.19_{\text {syst }}\right) \times 10^{-3}$, from which we derive $\operatorname{BR}(\phi \rightarrow$ $\left.\eta^{\prime} \gamma\right)=\left(6.20 \pm 0.11_{\text {stat }} \pm 0.25_{\text {syst }}\right) \times 10^{-5}$. Assuming the $\eta^{\prime}$ has zero gluonium content, we extract the pseudoscalar mixing angle in the quark-flavor basis, $\varphi_{P}=(41.4 \pm$ $\left.0.3_{\text {stat }} \pm 0.7_{\text {syst }} \pm 0.6_{\text {th }}\right)^{\circ}$. Combining the value of $R_{\phi}$ with other constraints, we estimate the fractional gluonium content of the $\eta^{\prime}$ to be $Z^{2}=0.14 \pm 0.04$ and the mixing angle to be $\varphi_{P}=(39.7 \pm 0.7)^{\circ}$.
\end{abstract}

Key words: $e^{+} e^{-}$collisions; radiative $\phi$ decays; pseudoscalar mixing angle PACS: 13.65.+i, 14.40.Aq

\section{Introduction}

The value of the $\eta-\eta^{\prime}$ mixing angle in the pseudoscalar meson nonet, $\theta_{P}$, has been discussed extensively over the last 35 years, and is today one of the most interesting SU(3)-breaking hadronic parameters to measure [1]. In the context of chiral perturbation theory, it has been demonstrated that a description of the $\eta-\eta^{\prime}$ system beyond leading order cannot be achieved in terms of just one mixing angle [2]. However, in the flavor basis the two mixing angles are equal, apart from terms which violate the OZI-rule; thus working in this basis it is still possible to use a single mixing angle, $\varphi_{P}$ [3]. The ratio $R_{\phi}$ of the two

1 Corresponding author.

E-mail address: camilla.didonato@na.infn.it (C. Di Donato). 
branching ratios $\phi \rightarrow \eta^{\prime} \gamma$ and $\phi \rightarrow \eta \gamma$ can be related to the $\eta-\eta^{\prime}$ mixing angle in the flavor basis $[3,4,5,6,7,8]$ and to the gluonium content of the $\eta^{\prime}$ meson $[5,9]$.

In this work we present a measurement of $R_{\phi}$ obtained using an integrated luminosity of $427 \mathrm{pb}^{-1}$ collected by KLOE during the years 2001-2002. The best measurement available to date was also published by KLOE [10], and was obtained from the analysis of the $\phi \rightarrow \eta^{\prime} \gamma$ decay with the $\pi^{+} \pi^{-} 3 \gamma$ final state and the $\phi \rightarrow \eta \gamma$ decay with the $\pi^{+} \pi^{-} \pi^{0} \gamma$ final state. The earlier KLOE measurement was based on an integrated luminosity of about $16 \mathrm{pb}^{-1}$, collected during the year 2000. Previous measurements were performed by the SND and CMD-2 collaborations $[11,12]$.

\section{DA $\Phi$ NE and KLOE}

The Frascati $\phi$ factory DA $\Phi$ NE is an $e^{+} e^{-}$collider running at a center-ofmass energy $\sqrt{s}=1.02 \mathrm{GeV}$, where $\phi$ mesons are produced with the beams colliding at a small crossing angle $(25 \mathrm{mrad})$. The KLOE detector consists of two main subdetectors: a large cylindrical drift chamber [13] and a sampling lead/scintillating-fiber electromagnetic calorimeter [14]. A superconducting coil surrounding the calorimeter provides a solenoidal field of $0.52 \mathrm{~T}$.

The drift chamber is $3.3 \mathrm{~m}$ in length and has a $2 \mathrm{~m}$ radius; it has full stereo geometry and operates with a gas mixture of $90 \%$ helium/10\% isobutane. The momentum resolution is $\sigma\left(p_{\perp}\right) /\left(p_{\perp}\right) \leq 0.4 \%$; the spatial resolution is $\sigma_{x y} \simeq 150 \mu \mathrm{m}$ and $\sigma_{z} \simeq 2 \mathrm{~mm}$. Vertices are reconstructed with a spatial resolution of $\simeq 3 \mathrm{~mm}$.

The calorimeter is divided into a barrel and two endcaps; it surrounds the drift chamber and covers $98 \%$ of the solid angle. The calorimeter is segmented into 2440 cells of cross section $4.4 \times 4.4 \mathrm{~cm}^{2}$ in the plane perpendicular to the fibers. Each cell is read out at both ends by photomultiplier tubes. Particle arrival times and the three-dimensional positions of the energy deposits are obtained from the signals collected at the two ends; cells close in time and space are grouped into a calorimeter cluster. The cluster energy $E$ is the sum of the cell energies, while the cluster time $T$ and position $R$ are energy-weighted averages. The probability for a photon to fragment into two or more clusters (splitting) is taken into account during event reconstruction by a dedicated procedure. The energy resolution is $\sigma_{E} / E=5.7 \% / \sqrt{E(\mathrm{GeV})}$; the timing resolution is $\sigma_{t}=57$

ps $/ \sqrt{E(\mathrm{GeV})} \oplus 100$ ps. The KLOE trigger [15] is based on calorimeter and chamber information. 


\section{Event selection}

The analysis has been performed using a sample of $\sim 1.4 \times 10^{9} \phi$ mesons collected in 2001-2002. We search for two categories of events. The first, $\phi \rightarrow$ $\eta^{\prime} \gamma \rightarrow \pi^{+} \pi^{-} 7 \gamma$, is produced through two different decay chains:

$$
\begin{aligned}
& \phi \rightarrow \eta^{\prime} \gamma \text { with } \eta^{\prime} \rightarrow \pi^{+} \pi^{-} \eta \text { and } \eta \rightarrow \pi^{0} \pi^{0} \pi^{0} \\
& \phi \rightarrow \eta^{\prime} \gamma \text { with } \eta^{\prime} \rightarrow \pi^{0} \pi^{0} \eta \text { and } \eta \rightarrow \pi^{+} \pi^{-} \pi^{0}
\end{aligned}
$$

The second, $\phi \rightarrow \eta \gamma \rightarrow 7 \gamma$, is produced through

$$
\phi \rightarrow \eta \gamma \text { with } \eta \rightarrow \pi^{0} \pi^{0} \pi^{0} .
$$

The latter channel is used for normalization of the rates and is practically background free [16]. Processes with $\phi \rightarrow K_{S} K_{L}$, where the $K_{L}$ decays near the beam interaction point (IP) can mimic the final state with $\pi^{+} \pi^{-} 7 \gamma$ because of the presence of an additional photon due either to machine background or split clusters. The most important decay chains of this type are the following:

$$
\begin{gathered}
\phi \rightarrow K_{S} K_{L} \text { with } K_{S} \rightarrow \pi^{+} \pi^{-} \text {and } K_{L} \rightarrow \pi^{0} \pi^{0} \pi^{0} \\
\phi \rightarrow K_{S} K_{L} \text { with } K_{S} \rightarrow \pi^{0} \pi^{0} \text { and } K_{L} \rightarrow \pi^{+} \pi^{-} \pi^{0} \\
\phi \rightarrow K_{S} K_{L} \text { with } K_{S} \rightarrow \pi^{+} \pi^{-} \gamma \text { and } K_{L} \rightarrow \pi^{0} \pi^{0} \pi^{0}
\end{gathered}
$$

In particular, the last of these channels has the same final state used to identify $\phi \rightarrow \eta^{\prime} \gamma$. Backgrounds from other sources are negligible.

All events must pass a first-level selection (FLS) [17], consisting of a machine background filter and an event selection procedure that assigns events into categories. After the FLS, we select $\phi \rightarrow \eta \gamma$ (normalization) events by requiring the presence of seven clusters not associated to tracks. Each cluster must have $E_{\gamma}>10 \mathrm{MeV}$ and polar angle $21^{\circ}<\theta_{\gamma}<159^{\circ}$ with respect to the beam direction, in order to exclude the focusing quadrupoles. Each cluster time must be compatible with the hypothesis of a photon coming from the IP, so that the requirement to be satisfied is $\left|T_{\gamma}-R_{\gamma} / c\right|<5 \sigma_{t}$, where $T_{\gamma}$ and $R_{\gamma}$ are the cluster time and position and $\sigma_{t}$ is the time resolution. We further require that no tracks come from the interaction region. To select $\phi \rightarrow \eta^{\prime} \gamma$ events, we similarly require seven prompt photons; in addition, we require the presence of a vertex formed by two tracks of opposite charge within a cylindrical region $\sqrt{x_{\mathrm{vtx}}^{2}+y_{\mathrm{vtx}}^{2}}<4 \mathrm{~cm}$ and $\left|z_{\mathrm{vtx}}\right|<8 \mathrm{~cm}$ around the IP. After applying these selections, we perform a kinematic fit requiring energy-momentum conservation, and times and path lengths to be consistent with the speed of light for photon candidates. We perform an inclusive measurement of the two different decay chains contributing to the $\phi \rightarrow \eta^{\prime} \gamma$ process.

The background is significantly reduced by the event-classification cuts in the 
FLS, which efficiently identify $K_{S}$ decays. By selecting events classified only as pure radiative decays, the $K_{S} K_{L}$ backgrounds are largely reduced. After this cut, background from processes (4) and (6) above is practically negligible, while to further reduce background from process (5), we require $E_{\gamma}>20 \mathrm{MeV}$ for each of the seven clusters, where the improved estimates of the cluster energies from the kinematic fit are used to evaluate this cut.

To estimate the absolute number of residual background events, the number of $\phi$ mesons in the sample has been evaluated. We obtain $N_{\phi}=(1.37 \pm 0.02) \times 10^{9}$, where the uncertainty comes from the estimate of the integrated luminosity and the experimentally-determined value of the leptonic decay width of the $\phi$. The luminosity is known with a total error of $0.6 \%$ [18], while the leptonic width has been measured by KLOE with a $1.7 \%$ uncertainty [19].

For each background channel, Table 1 lists the branching ratio multiplied by the overall selection efficiency as determined by Monte Carlo (MC) simulation. The branching ratio for process (6) has been evaluated using $\mathrm{BR}\left(K_{S} \rightarrow\right.$ $\left.\pi^{+} \pi^{-} \gamma\right)=(4.87 \pm 0.11) \times 10^{-3}$, for $E_{\gamma}>20 \mathrm{MeV} / c[20]$, the same cut used for our analysis.

Residual background due to multiphoton final states with a photon conversion and production of a charged-particle vertex has been reduced to a negligible level by a cut on the opening angle of the two tracks. Requiring $\cos \theta_{\pi^{+} \pi^{-}}<$ 0.84 [16] leads to a small effect on the signal efficiency and gives rise to a negligible contribution to the systematic error.

Table 1

Branching ratios times selection efficiencies $\mathrm{BR}\left(\phi \rightarrow K_{S} K_{L}\right) \cdot \mathrm{BR}\left(K_{S} \rightarrow \pi \pi(\gamma)\right) \cdot$ $\underline{\mathrm{BR}}\left(K_{L} \rightarrow \pi \pi \pi\right) \cdot \varepsilon(\mathrm{MC})$ and event yield for $\phi \rightarrow K_{S} K_{L}$ backgrounds

\begin{tabular}{lccc}
\hline$K_{S} / K_{L} \rightarrow$ & $\pi^{+} \pi^{-} / \pi^{0} \pi^{0} \pi^{0}$ & $\pi^{0} \pi^{0} / \pi^{+} \pi^{-} \pi^{0}$ & $\pi^{+} \pi^{-} \gamma / \pi^{0} \pi^{0} \pi^{0}$ \\
\hline $\mathrm{BR} \times \varepsilon(\mathrm{MC})$ & $(44 \pm 3) \cdot 10^{-9}$ & $(112 \pm 4) \cdot 10^{-9}$ & $(95 \pm 3) \cdot 10^{-9}$ \\
Background events & $60 \pm 4$ & $153 \pm 6$ & $130 \pm 5$ \\
\hline
\end{tabular}

After all selection cuts, we find 3750 events with an expected background of $N_{\text {background }}=343 \pm 43$. The background uncertainty comes from the uncertainties on the number of $\phi$ mesons, the selection efficiency $( \pm 15)$, and the evaluation of the machine background level $( \pm 28)[16]$. We treat the background uncertainty as a systematic contribution to the error on $N_{\eta^{\prime} \gamma}$. The final number of events from processes (1) and (2), after background subtraction, is $N_{\eta^{\prime} \gamma}=3407 \pm 61_{\text {stat }} \pm 43_{\text {syst }}$. The event-selection efficiency 2 after FLS cuts is

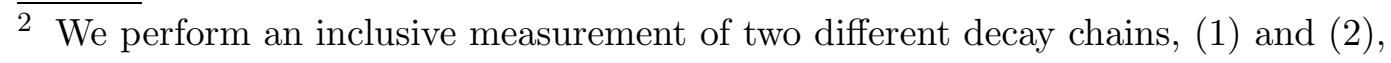


evaluated by $\mathrm{MC}$ simulation to be $\varepsilon_{\eta^{\prime}}=(23.45 \pm 0.16) \%$. The sample of MC signal events is five times larger than the data sample and has been produced by carefully simulating the run conditions during 2001-2002 data taking. Corrections for small data-MC discrepancies have been estimated directly from control data samples and applied. The FLS efficiency has been measured from data using a small subsample of minimum-bias events reconstructed without the FLS filters; the result is $\epsilon_{\eta^{\prime}}^{F L S}=(97 \pm 1) \%$.

Figure 1, left, shows the data-MC comparison of the photon-energy spectrum after all cuts. For $\eta^{\prime} \gamma$ events, the agreement is good. However, it is not possible to identify the recoil photon since its spectrum overlaps with the spectrum for photons from $\eta^{\prime}$ decay (see Fig. 1, right). Therefore, when building the $\pi^{+} \pi^{-} 6 \gamma$ invariant mass, we have a combinatorial background due to incorrect photon association. If we subtract the MC estimate of the physical and combinatorial background from data, we obtain a peak at the expected value of the $\eta^{\prime}$ mass, as seen in Fig. 2.
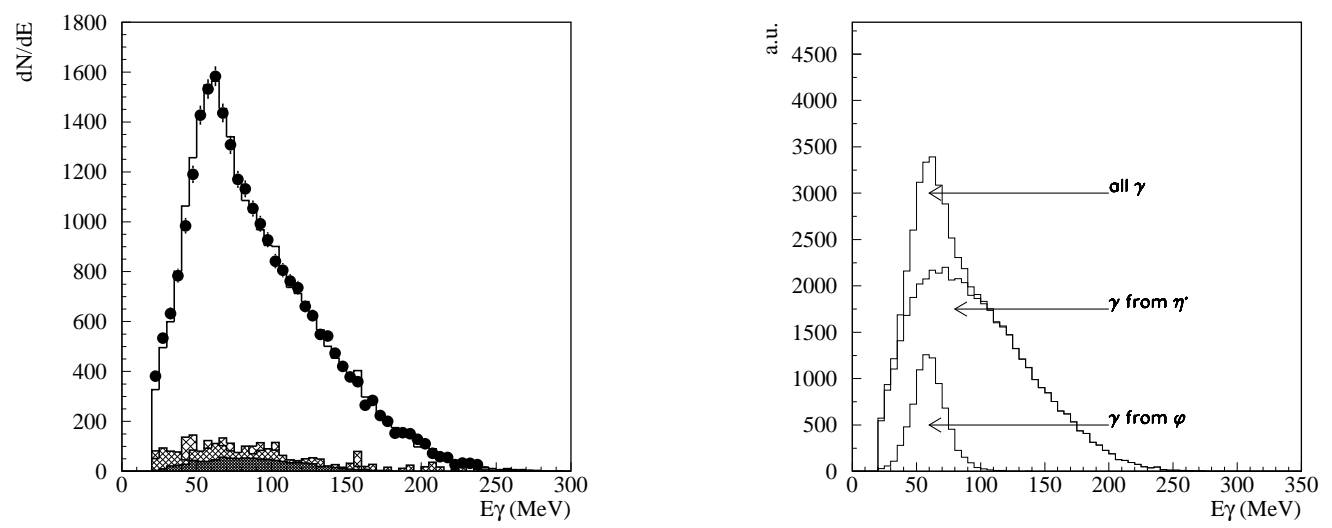

Fig. 1. $\phi \rightarrow \eta^{\prime} \gamma$. Energy distribution for all seven prompt neutral clusters. Left: Data-MC comparison after all cuts. Dots show data; histograms show MC distributions for each of the three background processes, (6), (5), and (4), and for the sum of signal and background contributions. Right: $\mathrm{MC} E_{\gamma}$ distributions for the recoil $\gamma$ emitted in the $\phi$ decay, the six $\gamma^{\prime}$ 's from the $\eta^{\prime}$ decay, and the overall distribution.

In contrast to the case of $\phi \rightarrow \eta^{\prime} \gamma$ decay, in $\phi \rightarrow \eta \gamma$ decay the recoil photon is the most energetic photon in the event. As a result, the recoil photons give rise to a distinct peak in the photon-energy spectrum. To select process (3), we additionally require the identification of the recoil photon with $320 \mathrm{MeV}<$ $E_{\gamma}<400 \mathrm{MeV}$. This cut is fully efficient for signal events and allows us to build

that contribute to the $\phi \rightarrow \eta^{\prime} \gamma$ process. The event-selection efficiency is obtained from the branching-ratio weighted average: $\varepsilon_{\eta^{\prime}}=\left(\varepsilon_{(1)} \mathrm{BR}_{(1)}+\varepsilon_{(2)} \mathrm{BR}_{(2)}\right) /\left(\mathrm{BR}_{(1)}+\right.$ $\left.\mathrm{BR}_{(2)}\right)$ 

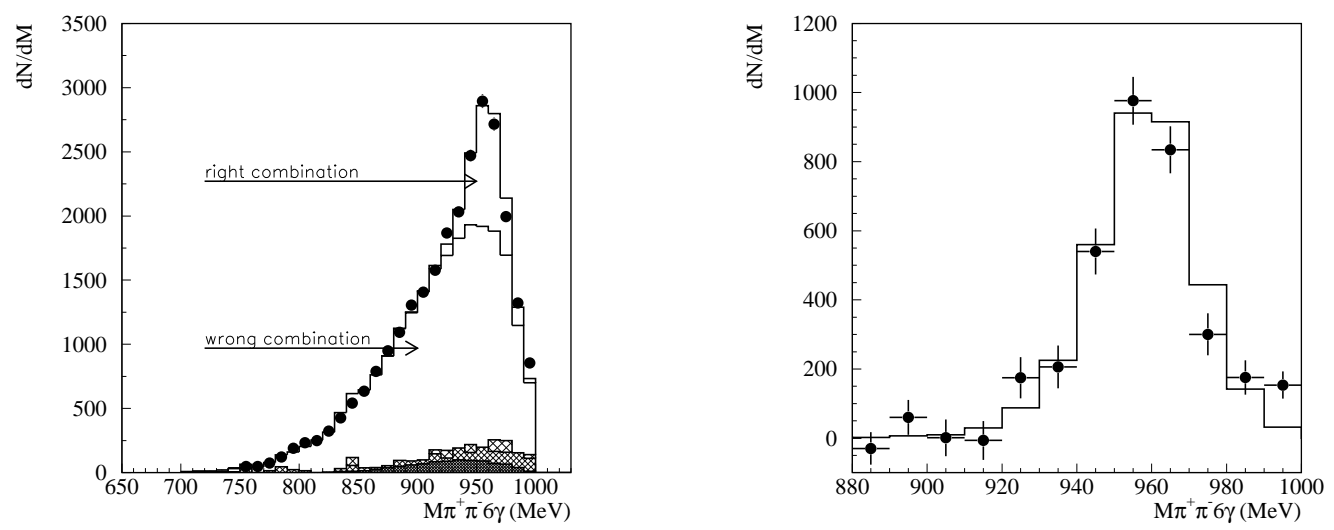

Fig. 2. $\phi \rightarrow \eta^{\prime} \gamma$. Left: Distribution of $M\left(\pi^{+} \pi^{-} 6 \gamma\right)$ for all possible combinations. Dots are data; the histogram represents the MC sum of the three background contributions, (6), (5) and (4), the combinatorial background, and the contribution from correctly paired signal events. Right: Data-MC comparison of $M\left(\pi^{+} \pi^{-} 6 \gamma\right)$ distributions in the area about the peak, after bin-by-bin subtraction.

the $6 \gamma$ invariant mass, as seen from Fig. 3. In all, we select $N_{\eta \gamma}=1665000$ events. The event selection efficiency after FLS is evaluated from MC; its value is $\varepsilon_{\eta}=(33.66 \pm 0.01) \%$. The FLS efficiency measured using data is $\epsilon_{\eta}^{F L S}=(97.88 \pm 0.10) \%$.
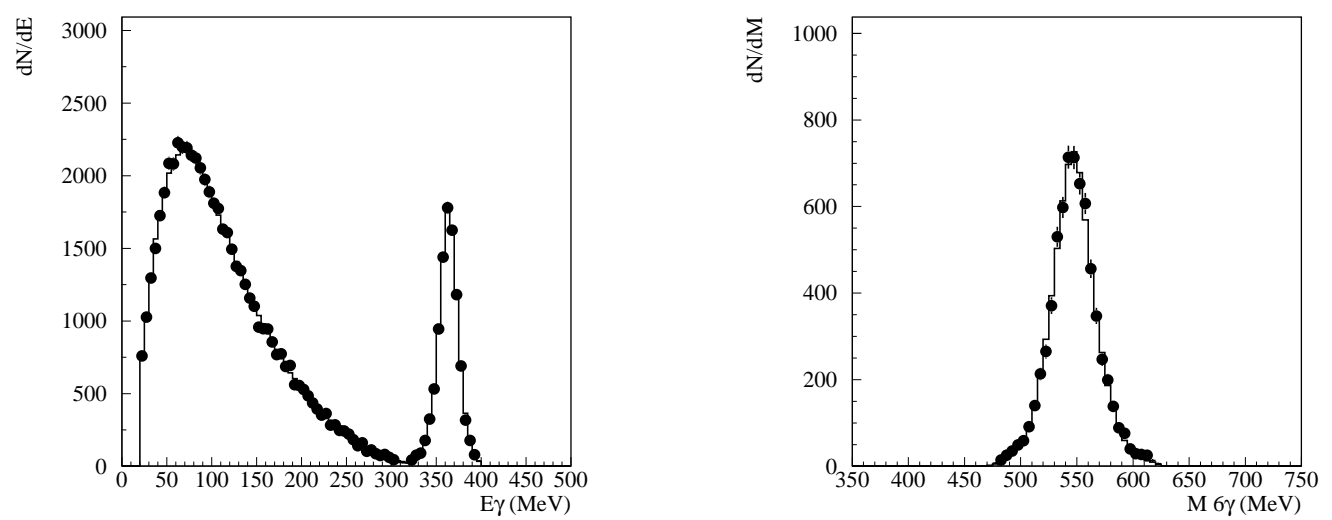

Fig. 3. $\phi \rightarrow \eta \gamma$. Left: Data-MC comparison of $E_{\gamma}$ distributions. Right: Data-MC comparison of $M(6 \gamma)$ distributions. In both panels, histogram shows MC; dots are data. 


\section{Results}

We evaluate the ratio of the two branching fractions $R_{\phi}=\mathrm{BR}\left(\phi \rightarrow \eta^{\prime} \gamma\right) / \mathrm{BR}(\phi \rightarrow$ $\eta \gamma$ ) using the relation

$$
R_{\phi}=\frac{N_{\eta^{\prime} \gamma}}{N_{\eta \gamma}} \frac{\varepsilon_{\eta}}{\varepsilon_{\eta^{\prime}}} \cdot C_{F L S} \cdot \frac{\mathrm{BR}_{\eta \gamma}}{\mathrm{BR}_{\eta^{\prime} \gamma}} \cdot K_{\rho}
$$

where $\mathrm{BR}_{\eta \gamma}$ is the branching fraction $\mathrm{BR}\left(\eta \rightarrow \pi^{0} \pi^{0} \pi^{0}\right)$ and $\mathrm{BR}_{\eta^{\prime} \gamma}$ is $\mathrm{BR}\left(\eta^{\prime} \rightarrow\right.$ $\left.\pi^{+} \pi^{-} \eta\right) \cdot \operatorname{BR}\left(\eta \rightarrow \pi^{0} \pi^{0} \pi^{0}\right)+\mathrm{BR}\left(\eta^{\prime} \rightarrow \pi^{0} \pi^{0} \eta\right) \cdot \operatorname{BR}\left(\eta \rightarrow \pi^{+} \pi^{-} \pi^{0}\right) ; \varepsilon_{\eta^{\prime}}$ and $\varepsilon_{\eta}$ are the overall selection efficiencies respectively for $\phi \rightarrow \eta^{\prime} \gamma$ and for $\phi \rightarrow \eta \gamma$; and $C_{F L S}=\epsilon_{\eta}^{F L S} / \epsilon_{\eta^{\prime}}^{F L S}$ is the ratio of the FLS efficiencies. The factor $K_{\rho}$ accounts for interference between the amplitudes $A\left(\phi \rightarrow \eta\left(\eta^{\prime}\right) \gamma\right)$ and $A\left(\rho \rightarrow \eta\left(\eta^{\prime}\right) \gamma\right)$, and has been evaluated in the Born approximation in the manner of Ref. [10]. This correction depends on $\sqrt{s}$, for which a mean value over run conditions has been evaluated; the standard deviation is taken as a contribution to the systematic uncertainty. The final value $K_{\rho}=0.95 \pm 0.01$ takes into account also radiative corrections to the $\phi$ cross section [19].

The systematic uncertainty on $R_{\phi}$ is dominated by the uncertainty on the intermediate branching ratios of the $\eta^{\prime}$. The evaluation of the systematic uncertainty on the ratio of analysis efficiencies is obtained by studying the samples and the stability of the results with respect to (i) the first level selection, (ii) the tracking and vertex efficiencies, and (iii) the cuts applied to select the final sample. The contribution to the uncertainty due to FLS comes from the uncertainty on the efficiency measured from data using a small subsample of minimum-bias events reconstructed from raw data without the FLS filters. The error due to the tracking and vertex efficiencies is estimated from the data-MC discrepancy observed for a control sample of $\phi \rightarrow \pi^{+} \pi^{-} \pi^{0}$ events with charged-pion momenta in the range covered by our analysis. A sizeable contribution to the uncertainty due to the final selection cuts comes from the inclusiveness of the cut on the $\chi^{2}$ probability from the kinematic fit, since the $\chi^{2} / N_{\text {dof }}$ distribution has a pronounced tail. We estimate this effect by moving the cut and evaluating the maximum variation of the ratio $\Delta(N / \epsilon) /(N / \epsilon)$ and its effect on $R_{\phi}$. The systematic error on $N_{\eta^{\prime} \gamma} / N_{\eta \gamma}$ comes from the background subtraction. All the contributions are summarized in Table 2.

The final result is:

$$
R_{\phi}=\left(4.77 \pm 0.09_{\text {stat }} \pm 0.19_{\text {syst }}\right) \times 10^{-3}
$$

Using the value of $\operatorname{BR}(\phi \rightarrow \eta \gamma)=(1.301 \pm 0.024) \%$ from the PDG [21] we find:

$$
\mathrm{BR}\left(\phi \rightarrow \eta^{\prime} \gamma\right)=\left(6.20 \pm 0.11_{\text {stat }} \pm 0.25_{\text {syst }}\right) \times 10^{-5}
$$


Table 2

Contributions to the systematic error on $R_{\phi}$

\begin{tabular}{lcl}
\hline Quantity with systematic source & Systematic error & Source \\
\hline \hline$N_{\eta^{\prime} \gamma} / N_{\eta \gamma}$ & $1.3 \%$ & Background \\
\hline$C_{F L S}$ & $1.0 \%$ & Preselection \\
\hline & $1.0 \%$ & Tracking efficiency \\
$\varepsilon_{\eta} / \varepsilon_{\eta^{\prime}}$ & $1.0 \%$ & Vertex efficiency \\
& $1.5 \%$ & Prob $\left(\chi^{2}\right)$ \\
\hline $\mathrm{BR}_{\eta \gamma} / \mathrm{BR}_{\eta^{\prime} \gamma}$ & $2.8 \%$ & \\
\hline$K_{\rho}$ & $1.0 \%$ & \\
\hline \hline Total & $4 \%$ & \\
\hline
\end{tabular}

The value of $R_{\phi}$ can be related to the pseudoscalar mixing angle. In the quarkflavor basis, the $\eta-\eta^{\prime}$ system can be parameterized in terms of just one angle:

$$
\begin{aligned}
|\eta\rangle & =\cos \varphi_{P}|q \bar{q}\rangle+\sin \varphi_{P}|s \bar{s}\rangle \\
\left|\eta^{\prime}\right\rangle & =-\sin \varphi_{P}|q \bar{q}\rangle+\cos \varphi_{P}|s \bar{s}\rangle .
\end{aligned}
$$

where $|q \bar{q}\rangle=(1 / \sqrt{2})|u \bar{u}+d \bar{d}\rangle$. Using the approach of Ref. [7,8], where $\mathrm{SU}(3)-$ breaking is taken into account via the constituent-quark mass ratio $m_{s} / \bar{m}, R_{\phi}$ is given by the following expression:

$$
R_{\phi}=\frac{\operatorname{BR}\left(\phi \rightarrow \eta^{\prime} \gamma\right)}{\operatorname{BR}(\phi \rightarrow \eta \gamma)}=\cot ^{2} \varphi_{P}\left(1-\frac{m_{s}}{\bar{m}} \frac{C_{N S}}{C_{S}} \frac{\tan \varphi_{V}}{\sin 2 \varphi_{P}}\right)^{2}\left(\frac{p_{\eta^{\prime}}}{p_{\eta}}\right)^{3}
$$

where $\varphi_{V}=3.4^{\circ}$ is the mixing angle for vector mesons, $p_{\eta\left(\eta^{\prime}\right)}$ is the $\eta\left(\eta^{\prime}\right)$ momentum in the $\phi$ center-of-mass, and the two parameters $C_{N S}$ and $C_{S}$ (see Table 3) represent the effect of the OZI-rule, which reduces the vector and pseudoscalar wave-function overlap [8]. From Eq. (10) we obtain the following result:

$$
\varphi_{P}=\left(41.4 \pm 0.3_{\text {stat }} \pm 0.7_{\text {syst }} \pm 0.6_{\text {th }}\right)^{\circ} .
$$

The theoretical uncertainty on the mixing angle has been evaluated from the maximum variation induced by the spread of the values for $m_{s} / \bar{m}, C_{N S}$, and $C_{S}$. In the traditional approach, $\eta-\eta^{\prime}$ mixing is parameterized in the octetsinglet basis; in this basis the value of the mixing angle becomes: $\theta_{P}=\varphi_{P}-$ $\arctan \sqrt{2}=\left(-13.3 \pm 0.3_{\text {stat }} \pm 0.7_{\text {syst }} \pm 0.6_{\mathrm{th}}\right)^{\circ}$.

In QCD, gluons may form a bound state, called gluonium, that can mix with neutral mesons. While the $\eta$ is well understood as an $\mathrm{SU}(3)$-flavor octet meson 
with a small singlet admixture, the $\eta^{\prime}$ is a good candidate to have a sizeable gluonium content. If we allow for non-zero $\eta^{\prime}$ gluonium content, we have the following parameterization:

$$
\left.\left|\eta^{\prime}\right\rangle=X_{\eta^{\prime}}|q \bar{q}\rangle+Y_{\eta^{\prime}}|s \bar{s}\rangle+Z_{\eta^{\prime}} \mid \text { gluons }\right\rangle
$$

where $Z_{\eta^{\prime}}$ parameterizes mixing with gluonium. Normalization implies $X_{\eta^{\prime}}^{2}+$ $Y_{\eta^{\prime}}^{2}+Z_{\eta^{\prime}}^{2}=1$ with

$$
\begin{aligned}
X_{\eta^{\prime}} & =\cos \phi_{G} \sin \varphi_{P} \\
Y_{\eta^{\prime}} & =\cos \phi_{G} \cos \varphi_{P} \\
Z_{\eta^{\prime}} & =\sin \phi_{G}
\end{aligned}
$$

where $\phi_{G}$ is the mixing angle for the gluonium contribution. Non-zero $\eta^{\prime}$ gluonium content would imply

$$
X_{\eta^{\prime}}^{2}+Y_{\eta^{\prime}}^{2}<1
$$

and Eq. (10) would have to be rewritten

$$
R_{\phi}=\cot ^{2} \varphi_{P} \cos ^{2} \phi_{G}\left(1-\frac{m_{s}}{\bar{m}} \frac{C_{N S}}{C_{S}} \frac{\tan \varphi_{V}}{\sin 2 \varphi_{P}}\right)^{2}\left(\frac{p_{\eta^{\prime}}}{p_{\eta}}\right)^{3}
$$

We may use $\mathrm{SU}(3)$ relations as proposed in Refs. $[5,8,9]$ to further constrain $X_{\eta^{\prime}}$ and $Y_{\eta^{\prime}}$. With the parameterization of Eq. (12), these relations may be written as:

$$
\begin{aligned}
\Gamma\left(\eta^{\prime} \rightarrow \gamma \gamma\right) / \Gamma\left(\pi^{0} \rightarrow \gamma \gamma\right) & =\frac{1}{9}\left(\frac{m_{\eta^{\prime}}}{m_{\pi}}\right)^{3}\left(5 \cos \phi_{G} \sin \varphi_{P}+\sqrt{2} \frac{f_{q}}{f_{s}} \cos \phi_{G} \cos \varphi_{P}\right)^{2} \\
\Gamma\left(\eta^{\prime} \rightarrow \rho \gamma\right) / \Gamma\left(\omega \rightarrow \pi^{0} \gamma\right) & =\frac{C_{N S}}{\cos \varphi_{V}} \cdot 3\left(\frac{m_{\eta^{\prime}}^{2}-m_{\rho}^{2}}{m_{\omega}^{2}-m_{\omega}^{2}} \frac{m_{\pi}}{m_{\eta^{\prime}}}\right)^{3} \cos ^{2} \phi_{G} \sin ^{2} \varphi_{P} \\
\Gamma\left(\eta^{\prime} \rightarrow \omega \gamma\right) / \Gamma\left(\omega \rightarrow \pi^{0} \gamma\right) & =\frac{1}{3}\left(\frac{m_{\eta^{\prime}}^{2}-m_{\omega}^{2}}{m_{\omega}^{2}-m_{\omega}^{2}} \frac{m_{\omega}}{m_{\eta^{\prime}}}\right)^{3}\left[C_{N S} \cdot \cos \phi_{G} \sin \varphi_{P}\right. \\
& \left.+2 \frac{m_{s}}{\bar{m}} C_{S} \cdot \tan \varphi_{V} \cdot \cos \phi_{G} \cos \varphi_{P}\right]^{2}
\end{aligned}
$$

Figure 4 shows the above constraints graphically in the $\left(X_{\eta^{\prime}}, Y_{\eta^{\prime}}\right)$ plane. The constraint from the KLOE measurement of $R_{\phi}$ is shown in the hypothesis of no $\eta^{\prime}$ gluonium content. To allow for gluonium, we have minimized a $\chi^{2}$ function with $\cos ^{2} \phi_{G}$ and $\cos ^{2} \varphi_{P}$ as free parameters by imposing the above constraints and including in the error matrix the uncertainties on the other parameters as shown in Table 3 . The values for $f_{q}$ and $f_{s}$ are from Ref. [3]; all other values are from [8]. We assume no correlations between the parameters.

The solution in the hypothesis of no gluonium content, i.e. $\cos ^{2} \phi_{G}=1$, yields 


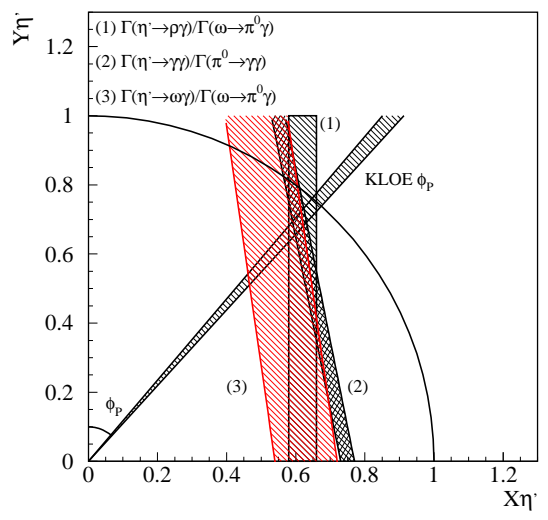

Fig. 4. Bounds on $X_{\eta^{\prime}}, \quad Y_{\eta^{\prime}}$. (1): $\Gamma\left(\eta^{\prime} \rightarrow \rho \gamma\right) / \Gamma\left(\omega \rightarrow \pi^{0} \gamma\right)$; $\quad(2)$ : $\Gamma\left(\eta^{\prime} \rightarrow \gamma \gamma\right) / \Gamma\left(\pi^{0} \rightarrow \gamma \gamma\right) ; \quad \operatorname{KLOE} \quad \varphi_{P}: \Gamma\left(\phi \rightarrow \eta^{\prime} \gamma\right) / \Gamma(\phi \rightarrow \eta \quad \eta \gamma) ; \quad(3):$ $\Gamma\left(\eta^{\prime} \rightarrow \omega \gamma\right) / \Gamma\left(\omega \rightarrow \pi^{0} \gamma\right)$

Table 3

Fixed parameters used in the fit for $\cos ^{2} \varphi_{G}$ and $\cos ^{2} \varphi_{P}$

\begin{tabular}{lccccc}
\hline Parameter & $f_{q}$ & $f_{s}$ & $C_{N S}$ & $C_{S}$ & $\frac{m_{s}}{\bar{m}}$ \\
\hline Value & $1 \pm 0.01$ & $1.4 \pm 0.014$ & $0.91 \pm 0.05$ & $0.89 \pm 0.07$ & $1.24 \pm 0.07$ \\
\hline
\end{tabular}

$\varphi_{P}=\left(41.5_{-0.7}^{+0.6}\right)^{\circ}$. The value of $\chi^{2} / N_{\text {dof }}$ is $11.34 / 3$, corresponding to a probability of 0.01 , and suggests a possible non-zero value for $Z_{\eta^{\prime}}^{2}$.

The solution allowing for gluonium is $\cos ^{2} \phi_{G}=0.86 \pm 0.04$ and $\cos ^{2} \varphi_{P}=$ $0.592 \pm 0.012$, from which $\varphi_{P}=(39.7 \pm 0.7)^{\circ}$ and $Z_{\eta^{\prime}}^{2}=0.14 \pm 0.04$, which means $\left|\varphi_{G}\right|=(22 \pm 3)^{\circ}$. In this case, $\chi^{2} / N_{\text {dof }}=1.42 / 2$, for a probability of 0.49 . When the constraint from $\eta^{\prime} \rightarrow \omega \gamma$ decay is relaxed, similar results are obtained: $\varphi_{P}=(39.8 \pm 0.8)^{\circ}$ and $Z_{\eta^{\prime}}^{2}=0.13 \pm 0.04$, while the $\chi^{2}$ probability improves to 0.97 . Figure 5 shows the four constraints in the $\left(Z_{\eta^{\prime}}^{2}, \varphi_{P}\right)$ plane, together with the allowed region for the solution with gluonium.

In the octet-singlet basis, the value of the $\eta-\eta^{\prime}$ mixing angle $\varphi_{P}=(39.7 \pm$ $0.7)^{\circ}$ becomes $\theta_{P}=(-15.0 \pm 0.7)^{\circ}$, which is in agreement with the value $\theta_{P}=(-15.9 \pm 1.2)^{\circ}$ measured by BES-II [22] using the branching fractions $B R\left(J / \psi \rightarrow \eta^{\prime} \gamma\right)$ and $B R(J / \psi \rightarrow \eta \gamma)$. These results for $\varphi_{P}$ are consistent with those coming from other processes as reviewed in Ref. [3], which quotes an average value $\varphi_{P}=(39.3 \pm 1.0)^{\circ}$. 


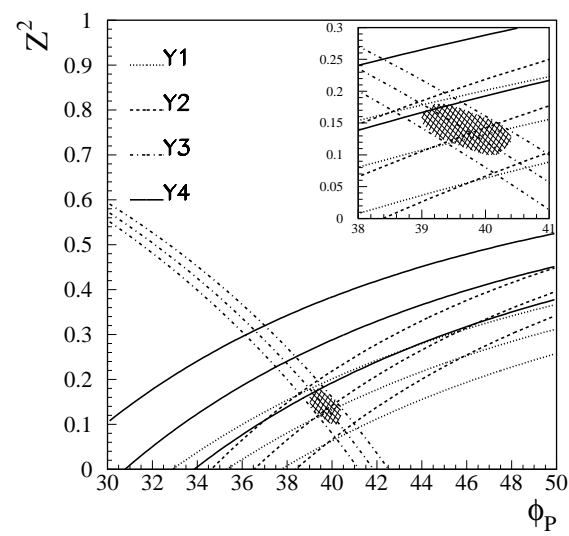

Fig. 5. The four constraints in the $\left(Z^{2}, \varphi_{P}\right)$ plane: $y_{1}=\Gamma\left(\eta^{\prime} \rightarrow \gamma \gamma\right) / \Gamma\left(\pi^{0} \rightarrow \gamma \gamma\right)$, $y_{2}=\Gamma\left(\eta^{\prime} \rightarrow \rho \gamma\right) / \Gamma\left(\omega \rightarrow \pi^{0} \gamma\right), y_{3}=R_{\phi}$, and $y_{4}=\Gamma\left(\eta^{\prime} \rightarrow \omega \gamma\right) / \Gamma\left(\omega \rightarrow \pi^{0} \gamma\right)$. The allowed region for the solution with gluonium is shown.

\section{Conclusion}

Analyzing about $1.4 \times 10^{9} \phi$ mesons collected by KLOE at the DA $\Phi$ NE collider, we have obtained a precise new measurement of the ratio $R_{\phi}=\mathrm{BR}(\phi \rightarrow$ $\left.\eta^{\prime} \gamma\right) / \operatorname{BR}(\phi \rightarrow \eta \gamma)=\left(4.77 \pm 0.09_{\text {stat }} \pm 0.19_{\text {syst }}\right) \times 10^{-3}$. From this measurement we obtain the most precise value of the pseudoscalar mixing angle in the flavor basis: $\varphi_{P}=\left(41.4 \pm 0.3_{\text {stat }} \pm 0.7_{\text {syst }} \pm 0.6_{\text {th }}\right)^{\circ}$. Allowing for gluonium in the $\eta^{\prime}$, a fit to our result together with other measurements yields $\varphi_{P}=(39.7 \pm 0.7)^{\circ}$ and a $\eta^{\prime}$ gluonium content of $Z_{\eta^{\prime}}^{2}=0.14 \pm 0.04$.

\section{Acknowledgements}

We thank the DA $\Phi$ NE team for their efforts in maintaining low background running conditions and their collaboration during all data taking. We want to thank our technical staff: G.F. Fortugno for his dedicated work to ensure an efficient operation of the KLOE Computing Center; M. Anelli for his continuous support of the gas system and the safety of the detector; A. Balla, M. Gatta, G. Corradi and G. Papalino for the maintenance of the electronics; M. Santoni, G. Paoluzzi and R. Rosellini for the general support of the detector; C. Piscitelli for his help during major maintenance periods. This work was supported in part by DOE grant DE-FG-02-97ER41027; by EURODAPHNE, contract FMRX-CT98-0169; by the German Federal Ministry of Education and Research (BMBF) contract 06-KA-957; by Graduiertenkolleg 'H.E. Phys. and Part. Astrophys.' of Deutsche Forschungsgemeinschaft, Con- 
tract No. GK 742; by INTAS, contracts 96-624, 99-37; and by the EU Integrated Infrastructure Initiative HadronPhysics Project under contract number RII3-CT-2004-506078.

\section{References}

[1] M. Benayoun et al., Phys. Rev. D 59 (1999) 114027.

[2] R. Kaiser and H. Leutwyler, hep-ph/9806336.

[3] T. Feldmann, Int. J. Mod. Phys. A 15 (2000) 159.

[4] C. Becchi and G. Morpurgo, Phys. Rev. B 687 (1965) 140.

[5] J.L. Rosner, Phys. Rev. D 27 (1983) 1101.

[6] P. Ball, J.M. Frere, M. Tytgat, Phys. Lett. B 365 (1996) 367.

[7] A. Bramon, R. Escribano and M.D. Scadron, Eur. Phys. J. C 7 (1999) 271.

[8] A. Bramon, R. Escribano and M.D. Scadron, Phys. Lett. B 503 (2001) 271.

[9] E. Kou, Phys. Rev. D 63 (2001) 54027.

[10] A. Aloisio et al., KLOE Collaboration, Phys. Lett. B 541 (2002) 45.

[11] V.M. Aulchenko et al., SND Collaboration, JETP 97 (2003) 24.

[12] R.R. Akhmetshin et al., CMD-2 Collaboration, Phys. Lett. B 473 (2000) 337; R.R. Akhmetshin et al., CMD-2 Collaboration, Phys. Lett. B 494 (2000) 26.

[13] M. Adinolfi et al., KLOE Collaboration, Nucl. Instrum. Methods A 488 (2002) 51.

[14] M. Adinolfi et al., KLOE Collaboration, Nucl. Instrum. Methods A 482 (2002) 364.

[15] M. Adinolfi et al., KLOE Collaboration, Nucl. Instrum. Methods A 492 (2002) 134.

[16] C. Di Donato, KLOE Note $214, \phi \rightarrow \eta^{\prime} \gamma$ in $\pi^{+} \pi^{-} 7 \gamma$ at KLOE, November 2006, http://www.lnf.infn.it/kloe/pub/knote/kn214.ps.

[17] F. Ambrosino et al., KLOE Collaboration, Nucl. Instrum. Methods A 534 (2004) 403.

[18] F. Ambrosino et al., KLOE Collaboration, Eur. Phys. J. C47 (2006) 589.

[19] F. Ambrosino et al., KLOE Collaboration, Phys. Lett. B 608 (2005) 199.

[20] E. Ramberg et al., Phys. Rev. Lett. 70 (1993) 2525.

[21] W.-M. Yao et al., J. Phys. G 33 (2006) 1.

[22] M. Ablikim et al., Phys. Rev. D 73 (2006) 052008. 